\title{
Panic of Buying Toilet Papers: A Historical Memory or a Horrible Truth? Systematic Review of Gastrointestinal Manifestations of COVID-19
}

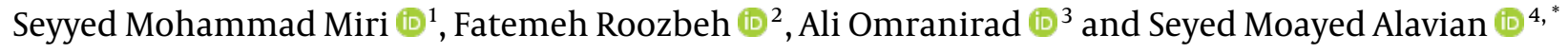 \\ ${ }^{1}$ Nedmedica, Heerlen, The Netherlands \\ ${ }^{2}$ Infectious Diseases Specialist, Mazandaran University of Medical Sciences, Sari, Iran \\ ${ }^{3}$ Anesthesiologist, Babol University of Medical Sciences, Babol, Iran \\ ${ }^{4}$ MELD Center, Tehran, Iran \\ "Corresponding author: MELD Center, Sepahbod Gharani Ave., Postal Code: 1598976511, Tehran, Iran. Tel: +98-2188945186, Email: alavian@thc.ir
}

Received 2020 March 15; Accepted 2020 March 15.

\begin{abstract}
Context: Within only 30 days after the first announcement of infection with "SARS-CoV-2" in Wuhan, it spread to more than 20 countries and become a pandemic by WHO as a "high alarming level of infection" with 136,343 involved cases all over the world.

Methods: To uncover more about the probable gastrointestinal transmission and its clinical manifestations, we reviewed all research publications with keywords including: “COVID-19”, “SARS-CoV-2”, “Coronavirus”, “outbreak”, “ARAS-CoV” in Medline, Scopus, Google Scholar as well as all news in social media about "novel COVID-19 pandemic". Out of 40 recent publications, 10 articles were studied by 2 authors.

Results: Although the SARS-CoV-2 tends to infect respiratory epithelial cells through the respiratory tracts, recent investigations detected the virus in the stool specimen, raising the question of the fecal-oral transmission route. Different studies showed that almost all gastrointestinal signs and symptoms were seen in elderly patients (more than 65 years old). including anorexia (40\%), nonspecific abdominal pains (8\%), diarrhea (10\%) and nausea and Vomiting in about $8 \%$ of patients. Anorexia and generalized abdominal pain suffer many treated cases even 10 - 20 days after negative blood tests (like CRP, lymphopenia) and negative PCR of respiratory samples. The main reason for this prolonged anorexia and abdominal pain may be due to the long persistence of COVID-19 in the gastrointestinal tracts after primary treatment.

Conclusions: Comparing to SARS, patients with COVID-19 showed less diarrhea, nausea, vomiting and/or abdominal discomfort before respiratory symptoms. Feces as a potential contagious source of Viral RNA can last even after viral clearance in the respiratory tract. We strongly recommend all cured patients of COVID-19 to disinfect and clean their toilets until 17-20 days after negative blood tests and solving their respiratory disorders. Gastrointestinal manifestations have seen in all men while no women referred with GI signs.
\end{abstract}

Keywords: COVID-19, Corona, Outbreak, SARS-CoV-2, Gastrointestinal

\section{Context}

Since early December 2019 which the most horrible virus agent titled "SARS-CoV-2" started to infect people from Wuhan, China, it only took less than 30 days to be spread to more than 20 countries all over the world. So that till 13 March 2020, the time of announcing COVID-19 as a pandemic by WHO and "high alarming level of infection" (1), 136,343 patients with COVID-19 were detected including 4,995 death and 70,437 recovered cases in more than 130 countries (2).

\section{Objectives}

Besides all images in the news, one strange capture became more astonishing: "The enthusiasm of buying toilet paper". The panic of buying lots of toilet paper was one of the first horrible and shocking videos spread all over the world within hours through social media even earlier than detecting the virus in the stool species. Is there any strong relationship between a respiratory virus (like SARS-CoV-2) and our gastrointestinal tracts? 


\section{Method}

We have reviewed all research publications with keywords including: "COVID-19”, "SARS-CoV-2", "Coronavirus", "outbreak”, "ARAS-CoV” in Medline, Scopus, Google Scholar as well as all news in social media about "novel COVID-19 pandemic". Out of 40 recent publications, 10 articles were studied over gastrointestinal manifestations which were included in our study by 2 authors. Why did coronavirus panic buy all of the toilet rolls?

\section{Results}

For the first time (2017), Zhou et al. found that intestinal pathways attend like an alternative infection route for eastern respiratory syndrome coronavirus (3). With the progression of the enteric infection, inflammation, viruspositive cells, and live viruses emerged in the lung tissues, indicating the development of sequential respiratory infection (3).

Principally, SARS-CoV-2 tends to infect respiratory epithelial cells through the respiratory tracts which lead to severe respiratory diseases. Some recent investigations detected the virus in the stool specimen, raising the question of the fecal-oral transmission route (4). For instance, in a study from China by Xinhua et al., they reported patients suffered only from diarrhea instead of classic viral symptoms like fever (5). Diarrhea symptoms may make the fecal-oral transmission of the virus more likely, especially if there is inadequate hand-washing or sanitary facilities. Furthermore, several previous studies about SARS revealed that the gastrointestinal tract (intestine) tropism of SARS coronavirus (SARS-CoV) was confirmed by the viral detection in biopsy specimens and stool even in discharged patients, which may partially provide explanations for the gastrointestinal symptoms, potential recurrence, and transmission of SARS from persistently shedding human as well (6). A recent non-reviewed study by Danchin et al. (7) also explained that both gut tropism and respiratory routes must be implemented to the benefit of epidemic control.

These recent studies could explain the coronavirus panic buy of all toilet rolls. On one hand, there are some psychological reasons for buying many toilet papers which may be related to the urgent and unnecessary need for washing hands or a sort of reflective reaction to watching images of panic buyers in other countries. On the other hand, it could be a consequence of gastrointestinal signs of COVID-19 like diarrhea or vomiting.
Finally, a non-reviewed study by Yaqian et al. (8) uncovered this fact: "people mixed up COVID-19 with its big brother SARS". While diarrhea was found in 30\% of patients with SARS, researchers found diarrhea in only $5 \%$ of patients with COVID-19. Similarly, fewer people with COVID19 had nausea and vomiting in comparison with SARS (6\% in COVID-19 vs. $27 \%$ in SARS). But how those people in the supermarkets reached this conclusion even before the first scientific reports? It seems that buying more toilet paper raised from a historical memory of SARS.

\subsection{Gastrointestinal Signs and Symptoms of COVID-19}

Despite all studies revealing the respiratory symptoms of COVID-19 such as fever, dry cough, dyspnea and clear evidence of droplet and contact transmission of SARS-CoV2 , others proposed a potential way of fecal-oral transmission $(5,7,9)$. First, the investigation by Zhou et al. (2017) (3) showed that MERS-CoV was significantly resistant to fed-state gastrointestinal fluids but less tolerant of highly acidic fasted-state gastric fluid. Then, the presence of virus (viral nucleic acids) in the fecal samples, as well as anal swabs of patients with COVID-19, was the first report by Zhang et al. (10) from the People's Hospital of Wuhan University. Till now, many studies confirmed the fact of oralfecal transmission. One of the key elements of a study by $\mathrm{Gu}$ et al. (11) was the permanent persistence of the virus in stool examination even 17 days after negative respiratory samples (17 out $73 \mathrm{pt}$ ) (9).

Based on these important findings, the disinfection of patients' vomitus, feces, and other bodily fluids is very important for both healthcare providers and family members of infected patients. One of the highly recommended hygienic advises for self-isolated patients could be the disinfection of toilets after each usage by COVID-19 patients.

Another important point was related to the order of respiratory vs. GI symptoms. Before the respiratory symptoms, many patients infected with COVID-19 came with diarrhea, nausea, vomiting and abdominal pain (11). Based on Xiao et al. (9) of the 73 hospitalized patients with SARSCoV-2, 39 patients ( 25 males and 14 females) tested positive for SARS-CoV-2 RNA in their stool (age ranged: 10 months to 78 years) even after 12 days of the first negative stool exam.

Through Medline search, we have found 6 different studies published between Dec 2019 and March 2020 about gastrointestinal manifestations of patients with COVID-19. Table 1 shows the percentage of different signs and symptoms in these studies.

Additionally, we have collected primary data of 100 suspected COVID-19 patients referred to a Corona clinic in Sari, Mazandaran in one week. The last row of Table 1 shows 


\begin{tabular}{|c|c|c|c|c|c|c|c|}
\hline & No. of Patients & Diarrhea & Nausea & Vomiting & Abdominal Pain & Haemoptysis & Anorexia \\
\hline Gao et al. (12) & & $2 \%-10.1 \%$ & $3 \%$ & $6 \%$ & & & \\
\hline Wang et al. (13) & 138 & $10 \%$ & $10 \%$ & $5 \%$ & $3 \%$ & & \\
\hline Chen et al. (14) & 99 & $2 \%$ & $1 \%$ & $1 \%$ & & & \\
\hline Xiao et al. (9) & 73 & $12 \%$ & & & & $25 \%$ & \\
\hline Huang et al. (15) & 41 & $3 \%$ & & & & $5 \%$ & \\
\hline Yaqian et al. (8) & & $5 \%$ & $6 \%$ & $6 \%$ & & & \\
\hline Our unpublished study & 100 & $10.2 \%$ & $7.6 \%$ & $7 \%$ & $8 \%$ & & $41 \%$ \\
\hline
\end{tabular}

our unpublished data which are in line with other similar studies from China. Almost all gastrointestinal signs and symptoms were seen in elderly patients (more than 65 years old). These young people with gastrointestinal symptoms were related to the complications of their medications like antiviral treatment regimens. Our clinical findings from Mazandaran (north of Iran) revealed that clinical signs and symptoms in suspected cases with COVID-19 are anorexia (40\%), nonspecific abdominal pains (8\%), diarrhea $(10 \%)$ and nausea and vomiting in about $8 \%$ of patients. Male preference was observed in our local study so that most of our patients with gastrointestinal manifestations were recorded in men.

Anorexia and generalized abdominal pain suffer many treated cases even 10 - 20 days after negative blood tests (like CRP, lymphopenia) and negative PCR of respiratory samples. The main reason for this prolonged anorexia and abdominal pain may be due to the long persistence of COVID-19 in the gastrointestinal tracts after primary treatment.

\subsection{Impaired Liver Functions Tests in COVID-19}

Laboratory findings in patients with COVID-19 demonstrated impaired liver function tests in a majority of cases. However, these nonspecific tests may rise in all inflammation like other viral infections. Few studies revealed several receptors to which different coronaviruses bind, such as angiotensin-converting enzyme 2 (ACE2) for SARS-CoV. Chai et al. (16) showed that specific ACE2 expression in cholangiocytes may cause liver damage after 2019-nCoV infection. The liver abnormalities of 2019-nCoV patients may not be due to hepatocyte damage, but cholangiocyte dysfunction. Therefore special care of liver dysfunction should be considered in treating 2019-nCoV patients during the hospitalization and shortly after cure (16).

\subsection{Probiotics Dairy Products Are Sold Out Completely}

Nowadays, another crisis was happened in supermarkets just by disappearing all dairy products labeled "probiotic" such as probiotic milk and yogurts. People rushed to buy probiotic products because they already knew about the effects of gut microbial agents on their appetites. To understand the mechanisms by which unbalanced dietary nutrients affect intestinal homeostasis, Hashimoto et al. (17) described ACE2 as a key regulator of dietary amino acid homeostasis, innate immunity, gut microbial ecology, and transmissible susceptibility to colitis. These results provide a molecular explanation for how amino acid malnutrition can cause intestinal inflammation and diarrhea. Respecting the similar pathogenesis of SARS and COVID-19, we proposed a relationship with gut microbiota.

\section{Conclusions}

Comparing to SARS, COVID-19 patients showed less diarrhea, nausea, vomiting and/or abdominal discomfort before respiratory symptoms. Feces as a potential contagious source of Viral RNA can last even after viral clearance in the respiratory tract. We strongly recommend all cured patients of COVID-19 to disinfect and clean their toilets until 17 - 20 days after negative blood tests and solving their respiratory disorders. Gastrointestinal manifestations have seen in all men while no women referred with GI signs.

\section{Footnotes}

Authors' Contribution: Seyed-Moayed Alavian proposed the idea and edited the final draft. Seyyed Mohammad Miri wrote the primary draft. Ali Omrani Rad collected the clinical data and edited the primary draft. Fatemeh Roozbeh added more clinical data and reviewed the literature and finalized the article. 
Conflict of Interests: Authors have no competing interest with the results of studies that are reviewed.

Ethical Approval: This is a review article based on other published articles and no need for any ethical approval.

Funding/Support: All financial matters are supported by the Publisher through an official invitation.

\section{References}

1. The Guardian. WHO declares coronavirus pandemic. 2020, [cited 133-2020]. Available from: https://www.theguardian.com/world/2020/ mar/11/who-declares-coronavirus-pandemic.

2. Worldmeter. COVID-19 coronavirus outbreak. 2020, [cited 13-3-2020]. Available from: https://www.worldometers.info/coronavirus/.

3. Zhou J, Li C, Zhao G, Chu H, Wang D, Yan HH, et al. Human intestinal tract serves as an alternative infection route for Middle East respiratory syndrome coronavirus. Sci Adv. 2017;3(11):eaao4966. doi: 10.1126/sciadv.aao4966. [PubMed: 29152574]. [PubMed Central: PMC5687858].

4. Kanne JP. Chest CT findings in 2019 novel coronavirus (2019-nCoV) infections from Wuhan, China: Key points for the radiologist. Radiology. 2020:200241. doi: 10.1148/radiol.2020200241. [PubMed:32017662].

5. The Star. New coronavirus could be transmitted through digestive system. 2020, [cited 13-3-2020]. Available from: https://www.thestar. com.my/news/regional/2020/02/02/new-coronavirus-could-betransmitted-through-digestive-system.

6. Healio Gastroenterology. Patients with COVID-19 may experience GI symptoms, possible fecal-oral transmission. 2020, [cited 15-32020]. Available from: https://www.healio.com/gastroenterology/ inflammatory-bowel-disease/news/online/\%7B991f3ae0-6f4d4211-93c6-b53bba5daf75\%7D/patients-with-covid-19-mayexperience-gi-symptoms-possible-fecal-oral-transmission.

7. Danchin A, Ng TWP, Turinici G. A new transmission route for the propagation of the SARS-CoV-2 coronavirus. medRxiv. 2020. doi: 10.1101/2020.02.14.20022939.
8. Yaqian M, Lin W, Wen J, Chen G. Epidemiological and clinical characteristics of SARS-CoV-2 and SARS-CoV: A system review. medRxiv. 2020 doi: 10.1101/2020.02.20.20025601

9. Xiao F, Tang M, Zheng X, Liu Y, Li X, Shan H. Evidence for gastrointestinal infection of SARS-CoV-2. Gastroenterology. 2020. doi: 10.1053/j.gastro.2020.02.055. [PubMed: 32142773].

10. Zhang W, Du RH, Li B, Zheng XS, Yang XL, Hu B, et al. Molecular and serological investigation of 2019-nCoV infected patients: Implication of multiple shedding routes. Emerg Microbes Infect. 2020;9(1):3869. doi: 10.1080/22221751.2020.1729071. [PubMed: 32065057]. [PubMed Central: PMC7048229].

11. Gu J, Han B, Wang J. COVID-19: Gastrointestinal manifestations and potential fecal-oral transmission. Gastroenterology. 2020. doi 10.1053/j.gastro.2020.02.054. [PubMed: 32142785].

12. Gao QY, Chen YX, Fang JY. 2019 Novel coronavirus infection and gastrointestinal tract.JDig Dis. 2020. doi: 10.1111/1751-2980.12851. [PubMed: 32096611].

13. Wang D, Hu B, Hu C, Zhu F, Liu X, Zhang J, et al. Clinical characteristics of 138 hospitalized patients with 2019 novel coronavirus-infected pneumonia in Wuhan, China. JAMA. 2020. doi: 10.1001/jama.2020.1585. [PubMed: 32031570]. [PubMed Central: PMC7042881].

14. Chen N, Zhou M, Dong X, Qu J, Gong F, Han Y, et al. Epidemiological and clinical characteristics of 99 cases of 2019 novel coronavirus pneumonia in Wuhan, China: A descriptive study. Lancet. 2020;395(10223):507-13. doi: 10.1016/S0140-6736(20)30211-7. [PubMed 32007143]

15. Huang C, Wang Y, Li X, Ren L, Zhao J, Hu Y, et al. Clinical features of patients infected with 2019 novel coronavirus in Wuhan, China. Lancet. 2020;395(10223):497-506. doi: 10.1016/S0140-6736(20)30183-5. [PubMed: 31986264].

16. Chai X, Hu L, Zhang Y, Han W, Lu Z, Ke A, et al. Specific ACE2 expression in cholangiocytes may cause liver damage after 2019-nCoV infection. bioRxiv. 2020. doi: 10.1101/2020.02.03.931766.

17. Hashimoto T, Perlot T, Rehman A, Trichereau J, Ishiguro H, Paolino M, et al. ACE2 links amino acid malnutrition to microbial ecology and intestinal inflammation. Nature. 2012;487(7408):477-81. doi: 10.1038/nature11228. [PubMed: 22837003] 